\title{
A SELF-ORGANIZING SCHEME FOR CONTROLLING THE MUSCLE RELAXATION PROCESS
}

\author{
Nabila M. El-Rabaie ; Hamdi A. Awad and Tarek A. Mahmoud \\ Faculty of Electronic Engineering, Menouf, 32952, Egypt \\ Awadhaa@yahoo.co.uk
}

(Received June 20, 2006 Accepted July 27, 2006)

\begin{abstract}
The main roles which are the concern of a clinical anaesthetist are those of drugs induced unconsciousness, muscle relaxation, and analgesia. The first two roles are concentrated in the operating theatre, while the third role is mainly concerned with postoperative conditions. Unlike, measurement of unconsciousness and analgesia the measurement of muscle relaxation process is considerable easier via monitoring of evoked electromyogram (EMG) signals. Among the features characterizing this process, time delay in initiation of muscle relaxation is perhaps the most challenging one. This time delay resulted from the drug circulation around the body and variation of the cardiac output. Another problem called nonlinearity mismatch that is resulted from the wide variability of identified models and their nonlinearity in the socalled pharmacodynamics for relaxant drugs behavior. This nonlinearity is due to the large inter-individual and intra-individual variability of the patient's parameters. These challenges can be treated with quantitative or qualitative techniques. The former was proved ineffective in trying to overcome these challenges. This paper proposes predictive self-organizing Auto Regressive eXogenous (PSO-ARX) scheme to deal with such challenges with ease. This is due to two notable features of the proposed scheme one is its plastic structure and the other is its small computation required compared with Generalized Predictive Control (GPC) schemes. Simulation results reflect the superiority of the proposed PSO-ARX scheme with respect to such schemes.
\end{abstract}

KEY WORDS: Neural networks, Medical systems, Self-organizing controllers

\section{INTRODUCTION}

Anaesthesia is the science of removing sensation of, and reaction to, a surgical operation. Modern general anaesthesia comprises unconsciousness (or depth of anaesthesia), muscle relaxation (or paralysis), and analgesia (or pain relief). The first two operations are concentrated in the operating theatre, whereas the third operation is mainly concerned with post-operative condition [1]. Each of these operations has been considered in recent years as possible scenarios for automated drug infusion via 
feedback control strategy. The main problem in the drug-induced depth of anaesthesia and muscle relaxation is the measurement of the clinical signs, which can be used online to the controller [2]. Depth of anesthesia is hard to define and hence to measure accurately, while, the measurement of the muscle relaxation level is considerable easier. A common approach of the measurement of the muscle relaxation is the monitoring of evoked electromyogram (EMG) signals produced at the hand via stimulation above the wrist. This stimulation ensures that all the nerve fibers are recruited, while suitable processing of the resultant EMG provides an analogue signal inversely proportional to the level of relaxation [3].

Among the features characterizing biomedical systems, time delay is perhaps the most challenging one. Two sources of such delay, one is the drug concentration around the body and the other is the variation of the cardiac output. Designing controllers to overcome time delays has always presented a serious challenge for engineers. Also this challenge grows even bigger when the value of this time delay is unknown or is prove to variations. PID controllers, whose benefits are still generally utilized within industry, can prove ineffective in trying to overcome this problem [4]. Smith predictor [5] has been shown to be very advantageous; however its performance may deteriorate considerably in the presence of a large process mismatch. That has always been one of the major problems in this paper. Generalized Predictive Control (GPC) schemes have also proposed to deal with challenges [6], however their performance can prone also ineffective in trying to overcome these challenges. Self-organizing techniques are promising ones to deal with such circumstances. Accordingly, this paper proposes Predictive Self-Organizing Auto Regressive-eXogenous (PSO-ARX) scheme to overcome such challenges easily.

This paper can be organized as follows. Section 2 formulates our problem. It describes the mathematical model of the muscle relaxation process and the main characteristics of this process. Section 3 describes the proposed PSO-ARX scheme. Section 4 depicts simulation results using the proposed scheme. Section 5 concludes the topics discussed in this paper.

\section{THE PROBLEM FORMULATION}

To facilitate the design of advanced controllers, it is necessary to have a good mathematical model of the process. In order to identify the muscle relaxation process associated with drugs, pharmacological modelling is commonly used to describe the metabolism of such drugs. The pharmacological modelling comprises two main categories known as pharmacokinetics and pharmacodynamics. The former studies the relationship that exists between drug dose and drug concentration in the blood plasma. Interpretation of this relationship can give a mathematical meaning via the concept of compartmental models. While, the latter concerns with the drug concentration and the effect produced. One of the common drugs used in this operation is the atracurium drug, which is a non-depolarising fact acting agent and has gained popularity over pancuronium and d-tubocurarine [3]. 


\section{2-1. Pharmacokinetics}

Basically, the pharmacokinetics studies what the body does to the drug. In other words, after the drug injection, the plasma concentration of atracurium declines rapidly in two exponential phases corresponding to distribution and elimination [7]. Therefore, a conventional two-compartmental model is used by adding an elimination path from the peripheral compartmental as depicted in Fig.1. Suppose $x_{i}$ is the drug concentration at time $\mathrm{t}, \dot{x}_{i}$ its rate of change, and $u$ is the drug input, then:

$$
\begin{aligned}
& \dot{x}_{1}=-\left(k_{10}+k_{12}\right) x_{1}+k_{21} x_{2}+u \\
& \dot{x}_{2}=k_{12} x_{1}-\left(k_{20}+k_{21}\right) x_{2}
\end{aligned}
$$

Using Laplace transform, equation (1) can be rewritten as:

$$
\begin{aligned}
& s X_{1}=-\left(k_{10}+k_{12}\right) X_{1}+k_{21} X_{2}+U \\
& s X_{2}=k_{12} X_{1}-\left(k_{20}+k_{21}\right) X_{2} \\
& \frac{X_{1}(s)}{U(s)}=\frac{s+k_{20}+k_{21}}{\left(s+k_{10}+k_{12}\right)\left(s+k_{20}+k_{21}\right)-k_{12} k_{21}}
\end{aligned}
$$

The mean values for the pharmacokinetics parameters are [3]:

$k_{12}+k_{10}=0.26 \mathrm{~min}^{-1}$

$k_{21}+k_{20}=0.094 \mathrm{~min}^{-1}$

$k_{12} k_{21}=0.015 \mathrm{~min}^{-2}$

Substituting in equation (3) leads to:

$$
\frac{X_{1}(s)}{U(s)}=\frac{9.94(1+10.64 s)}{(1+3.08 s)(1+34.42 s)}
$$

which describes the pharmacokinetics of the muscle relaxation system relating to the drug atracurium in a transfer function.

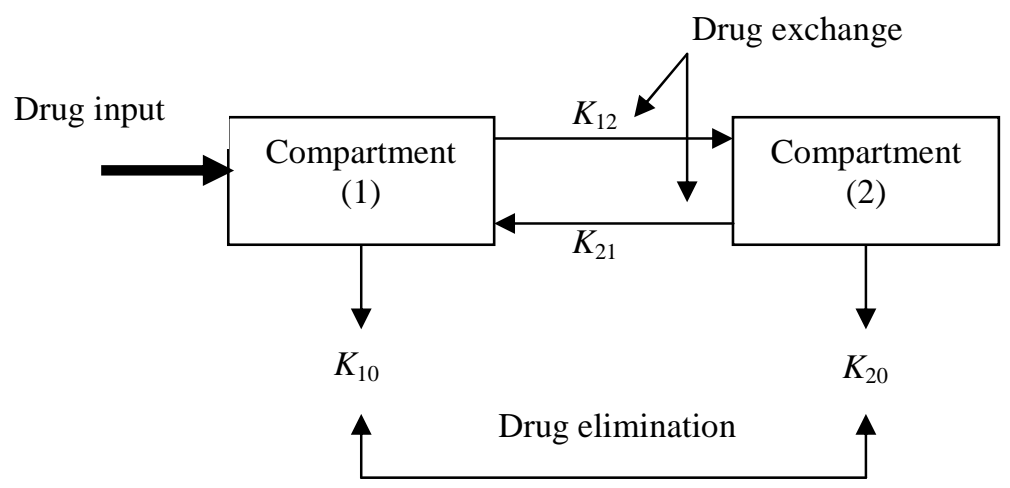

Fig. 1: A two compartmental model for atracurium drug. 


\section{2-2. Pharmacodynamics}

In this phase, to identify the drug effect, a third compartment known as the "effect compartment" has been introduced to the atracurium kinetics. It is connected to the central compartment by a first-order rate constant $k_{I E}$, whereas the rate constant $k_{E O}$ characterises the drug dissipation from the effect compartment, as depicted in Fig. 2. In this latter compartment, the drug concentration change is governed by the following equation:

$$
\dot{x}_{E}=k_{1 E} x_{1}-k_{E 0} x_{E}
$$

Using Laplace transforms yields:

$$
X_{E}(s)=\frac{K_{1 E} X_{1}(s)}{s+K_{E 0}}
$$

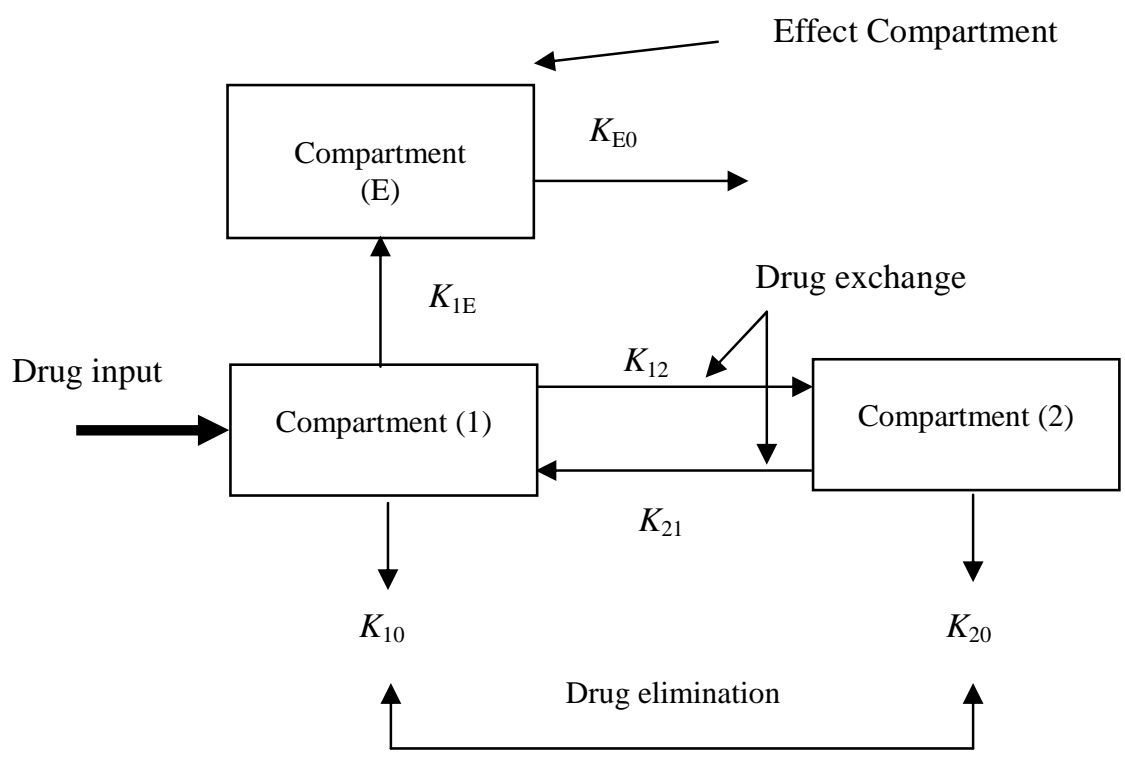

Fig. 2: Modification of the atracurium kinetics.

The Hill equation [7-9] may be used to relate the effect to a specific blood concentration of drug:

$$
E_{\text {eff }}=\frac{E_{\max }}{1+\frac{X_{E}(50)^{\alpha}}{X_{E}^{\alpha}}}
$$

where $E_{\text {eff }}, E_{\max }$ and $X_{E}(50)$ are the drug effect produced (paralysis), the maximum drug effect ( $100 \%$ paralysis), and the drug concentration of at $50 \%$ effect. The mean values of the pharmacodynamics are [3]: 
$k_{E 0}=0.208 \mathrm{~min}^{-1}$,

$X_{E}(50)=0.404 \mu g m l^{-1}$,

$\alpha=2.98$,

$k_{1 E}=10^{-4} \mathrm{~min}^{-1}$

Combining equations (6) and (4) and normalizing the open loop gain at 1.0 leads to:

$$
\frac{X_{E}}{U}=\frac{k\left(1+T_{4} s\right) e^{-\tau s}}{\left(1+T_{1} s\right)\left(1+T_{2} s\right)\left(1+T_{3} s\right)}
$$

where $K=1.0, T_{1}=4.81 \mathrm{~min}, T_{2}=34.42, T_{3}=3.08 \mathrm{~min}, T_{4}=10.64 \mathrm{~min}$ and $\tau=1 \mathrm{~min}$. Finally, the overall nonlinear model obtained by combining equation (8) together with Hill equation (7) as depicted in Fig. 3.

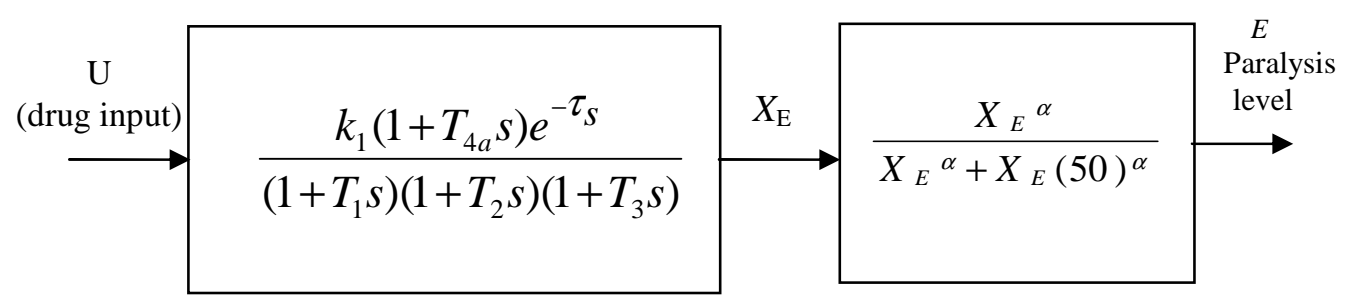

Fig. 3: Nonlinear model of the muscle relaxation process.

Figure 4 shows a series of Hill equations for different parameters of $\alpha$ and $X_{E}(50)$. For nominal Hill equation values, a linearized gain for operating points ranges from 0.85 to 0.95 for paralysis can lead to difficulties due to the curved shapes around this region. Also, the patient-to-patient parameter variability can affect the nonlinearity shape (uncertainty) by making it steeper or more flat. All these considerations make the muscle relaxation process a very challenging one. Time delay, nonlinearity mismatch, and uncertainties of such process are challenges problem have to be overcome.

\section{THE PROPOSED PSO-ARX CONTROL SCHEME}

The schematic diagram of the proposed PSO-ARX control scheme is shown in Fig. 5. At instance $k$, the ARX Local Model (ARX-LM) -based predictive controller predicts the controller parameters of the controller, then the controller update it self to generate the optimal control signal. The controller send this signal to the process and its output is compared with the ARX-LM network to estimate another set of parameters and then the controller repeat its function.

The self-orgnizing ARX-LM network plays an important role in this scheme to deal with the challenges mentioned in section 2 . The following subsection describes briefly the structure and the learning phases of the employed network [10]. 


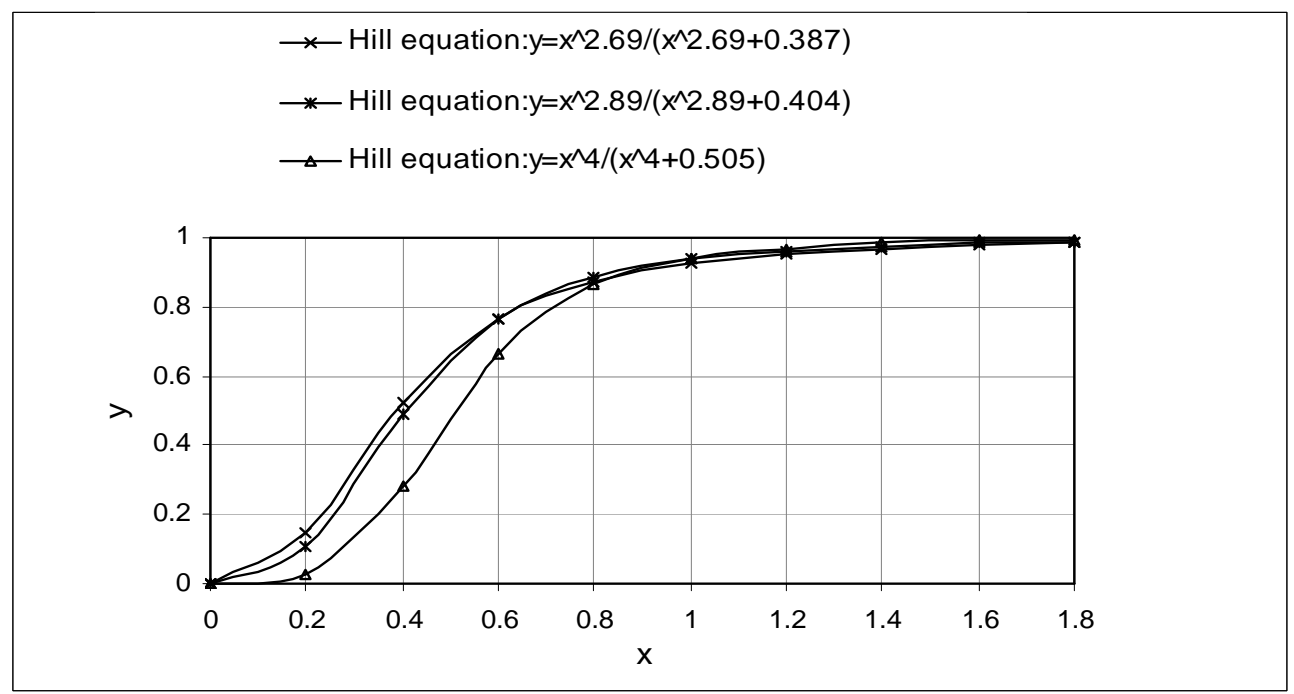

Fig. 4: Graph showing the shape of the various nonlinearity curves used in the process model.

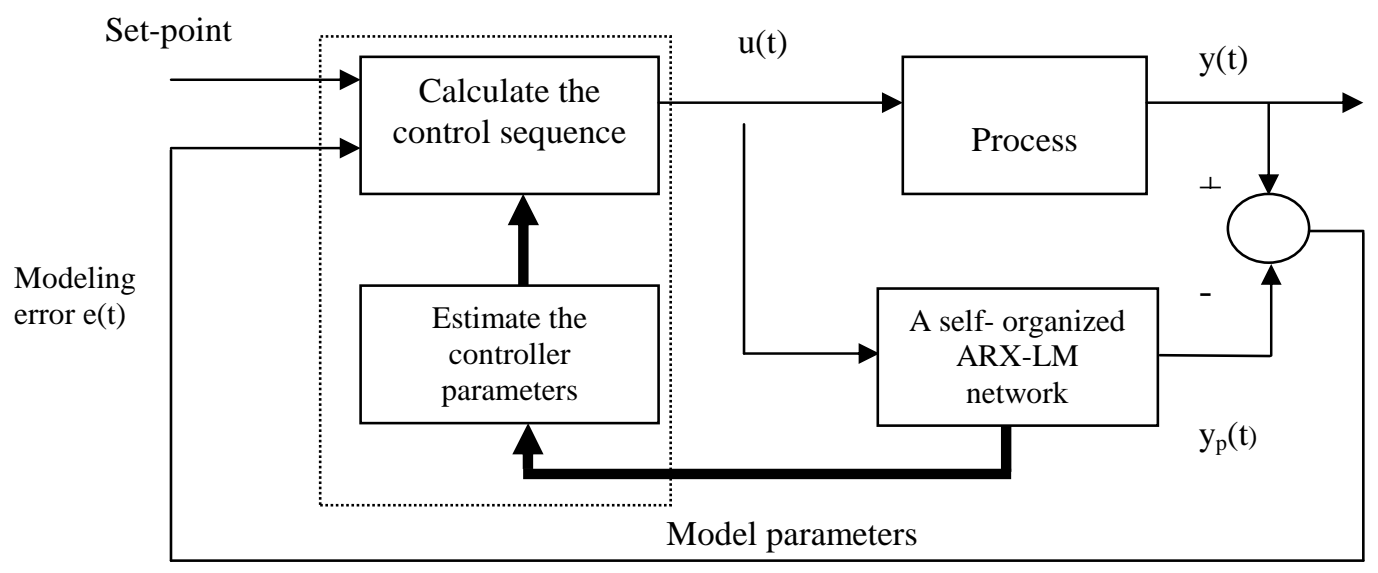

Fig. 5: The proposed PSO-ARX control scheme.

\section{3-1. The Structure of the Self-Organized ARX-LM Network}

This network consists of a set of TSK fuzzy rules [11] fertilized by wavelet functions [12]. Each wavelet determines the contribution of the corresponding TSK fuzzy model. These sub-models are merged to generate the final output of the proposed network. It consists of five layers as depicted in Fig. 6 that can be described as follows:

Layer -1: A node at this layer just transmits the input values to the next layer.

Layer -2: This layer consists of two groups, universes of discourse of the input fuzzy variables and their wavelets. The former cover the universes of discourse of the input variables by a set of triangular-shipped function $\mu_{A_{j}^{i}}\left(x_{i}\right)$. That is: 


$$
\mu_{A_{j}^{i}}\left(x_{i}\right)=1-\frac{2\left|x_{i}-c_{i j}\right|}{\delta_{i j}}
$$

where $A_{j}^{i}$ is the $\mathrm{j}^{\text {th }}$ fuzzy set of the $\mathrm{i}^{\text {th }}$ input variable $x_{i}$, and $c_{i j}$, and $\delta_{i j}$ are the center and width of this fuzzy set.

The latter is a wavelet function generated by dilating and translating the mother wavelet function $h(x)=\left(1-x^{2}\right) \exp \left(-\frac{x^{2}}{2}\right)$. That is :

$$
\Phi_{j}(X)=\prod_{k=1}^{n} h\left(Z_{j k}\right)
$$

where $Z_{j k}=\frac{x_{k}-m_{j k}}{d_{j k}}, n$ is the number of their inputs, $m$ and $d$ are the translation and dilation parameters respectively.

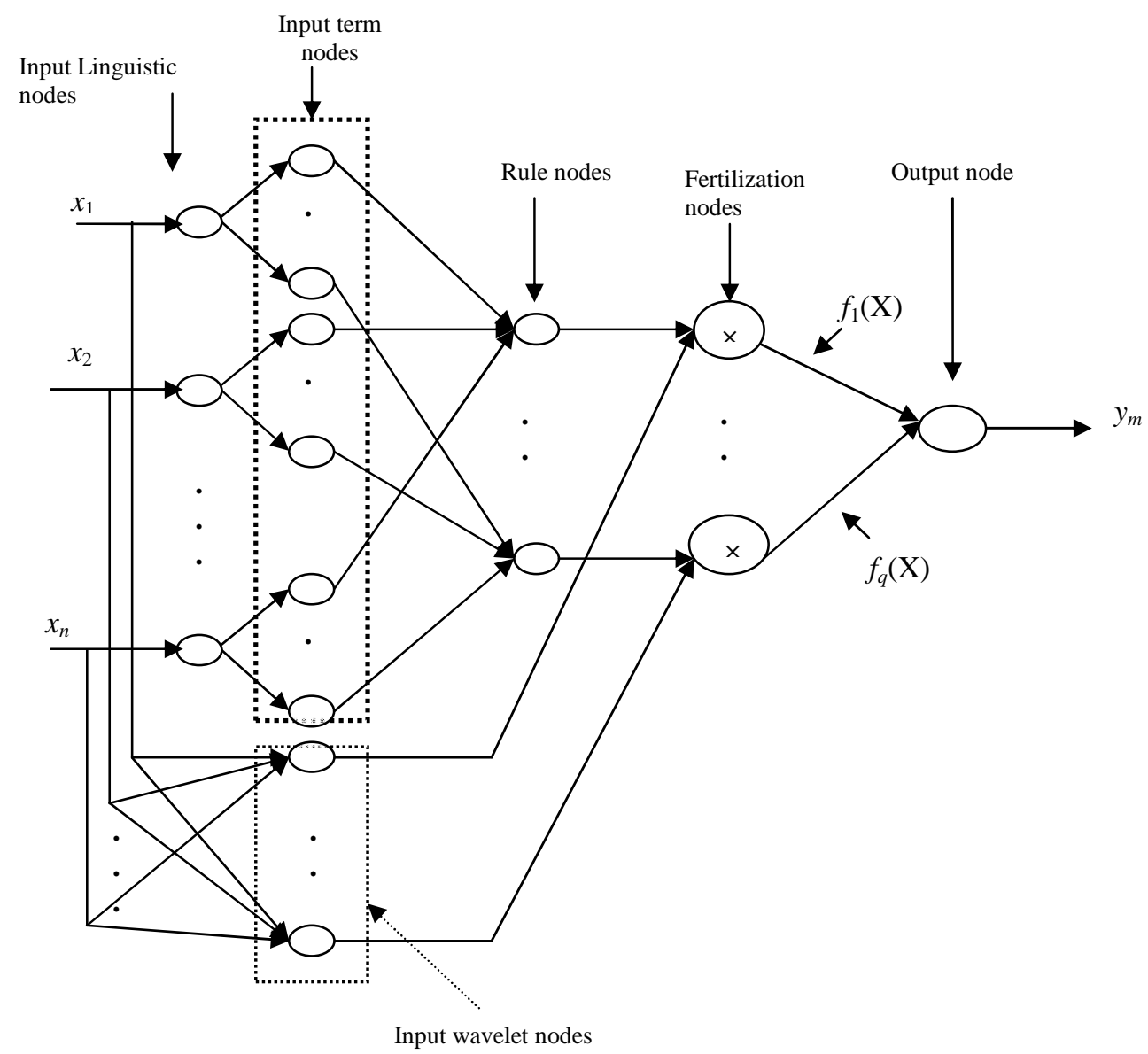

Fig. 6: The structure of the self-organized ARX-LM network. 
Layer -3: The firing strength can be obtained using Larsen's product [13] as follows:

$$
\omega^{i}=\prod_{j=1}^{n} \mu_{A_{j}^{i}}\left(x_{i}\right)
$$

where, its normalized value is:

$$
\varpi^{i}=\frac{\omega^{i}}{\sum_{i=1}^{q} \omega^{i}}
$$

Layer -4: A node at this layer is a sub-model that merges the normalized firing strength of a TSK fuzzy rule with a wavelet. That is:

$$
y_{i}{ }^{4}=\Phi_{i}(X) \varpi^{i}
$$

Layer -5: Based on the approximate Center Of Area (COA) defuzzification method, the crisp output $y_{m}$ can be deduced. That is:

$$
y_{m}=\sum_{i=1}^{q} \varpi^{i} f_{i}(\mathrm{X}) \Phi_{i}(X)
$$

The network described above performs the following rule:

$$
R^{i} \text { : IF } x_{1} \text { is } A_{1}{ }^{i} \text { and } \ldots \text { and } x_{n} \text { is } A_{n}{ }^{i} \text { THEN } y^{i}=f_{i}(\mathrm{X}) * \Phi_{i}(X)
$$

where,

$f_{i}(X)$ is a linear function of the TSK model. That is:

$$
f_{i}(X)=w_{1}^{i} * x_{1}+w_{2}^{i} * x_{2}+. \quad . \quad+w_{1}^{i} * x_{n}
$$

and, $\Phi_{i}(X)$ is the wavelet function defined in (10).

Reforming (16), results an ARX-LM defined below:

$$
f_{i}(X)=w_{u 1}^{i} * u(k-1)+\ldots .+w_{u r}^{i} * u(k-r)+w_{y 1}^{i} * y(k-1)+\ldots .+w_{y s}^{i} * y(k-s)
$$

Substituting (17) in (14), results:

$$
y_{m}(k)=b_{1} * u(k-1)+. . .+b_{r} * u(k-r)+a_{1} * y(k-1)+\ldots .+a_{s} * y(k-s)
$$

where,

$$
b_{i}=\sum_{j=1}^{q} w_{u i}^{j} * \Phi_{j}(X) \varpi^{j}, i=1,2, \ldots r, \quad a_{h}=\sum_{j=1}^{q} w_{y h}^{j} * \Phi_{j}(X) \varpi^{j}
$$

$h=1,2, \ldots, s$ and $q$ is the number of rules generated, and $r$ and $s$ are the orders of the plant input and output respectively. Equation (18) represents the ARX-LM with the input and output vectors defined below: 
$\underline{X}=[\mathrm{u}(\mathrm{k}-1) \mathrm{u}(\mathrm{k}-2) \ldots \mathrm{u}(\mathrm{k}-\mathrm{r}) \mathrm{y}(\mathrm{k}-1) \mathrm{y}(\mathrm{k}-2) \ldots, . \mathrm{y}(\mathrm{k}-\mathrm{s})]^{\mathrm{T}}$ and $\underline{Y}=[\mathrm{y}(\mathrm{k})]^{\mathrm{T}}$

Learning of the ARX-LM network consists of two phases, structure learning and parameter learning. The former structure is the corner stone to develop an optimal ARX-LM network. It should determine the optimal number of the fuzzy clusters of each fuzzy variable, the wavelet nodes, and the fuzzy rules. The fuzzy ART algorithm [14] was employed to determine the above three parameters. The RLS method was employed in the latter learning phase of this network. Employing the ART and the RLS algorithms to develop ARX-LM network, resulted a simple self-organizing network for modeling and control dynamic systems.

\section{3-2. The Proposed PSO-ARX Control Scheme}

The basic idea behind the proposed algorithm is that its model structure can instantaneously be self-organized to deal with the challenges mentioned in section 2 . To clarify this idea, consider a single-input and single-output discrete time system whose model prediction over the costing horizon time $n_{2}$ is given by:

$\mathrm{y}_{\mathrm{p}}(\mathrm{t}+1)=\mathrm{a}_{1} \mathrm{y}(\mathrm{t})+\ldots+\mathrm{a}_{\mathrm{s}} \mathrm{y}(\mathrm{t}+1-\mathrm{s})+\mathrm{b}_{1} \mathrm{u}(\mathrm{t})+\ldots+\mathrm{b}_{\mathrm{r}} \mathrm{u}(\mathrm{t}-\mathrm{r}+1)+\mathrm{e}(\mathrm{t})$

$\mathrm{y}_{\mathrm{p}}(\mathrm{t}+i)=\mathrm{a}_{1} \mathrm{y}(\mathrm{t}+i-1)+\ldots+\mathrm{a}_{\mathrm{s}} \mathrm{y}(\mathrm{t}+i-\mathrm{s})+\mathrm{b}_{1} \mathrm{u}(\mathrm{t}+i)+\ldots+\mathrm{b}_{\mathrm{r}} \mathrm{u}(\mathrm{t}+i-\mathrm{r})+\mathrm{e}(\mathrm{t})$

$\mathrm{y}_{\mathrm{p}}\left(\mathrm{t}+n_{2}\right)=\mathrm{a}_{1} \mathrm{y}\left(\mathrm{t}+n_{2}-1\right)+\ldots+\mathrm{a}_{\mathrm{s}} \mathrm{y}\left(\mathrm{t}+n_{2}-\mathrm{s}\right)+\mathrm{b}_{1} \mathrm{u}\left(\mathrm{t}+n_{2}-1\right)+\ldots+\mathrm{b}_{\mathrm{r}} \mathrm{u}\left(\mathrm{t}-\mathrm{r}+n_{2}\right)+\mathrm{e}(\mathrm{t})$

where e $(t)$ represents the modeling error, and $y_{p}(t+\mathrm{i})$ is the $i^{\text {th }}$ predicted output. It has been assumed that the modeling error is constant over the entire prediction horizon and the values of $\mathrm{u}(t+m-1)$ is equal zero over the control horizon $m$. Accordingly, the above equations can be reformed as follows:

$$
Y(t)=P X(t)+Q U(t)+R e(t)
$$

where, $\mathrm{Y}(\mathrm{t})=\left[y_{p}(t+1) \ldots y_{p}\left(t+n_{2}\right)\right]^{\mathrm{T}}$, denotes a vector of the model predicted outputs over the prediction horizon, $\mathrm{X}(\mathrm{t})=[\mathrm{y}(\mathrm{t}) \mathrm{y}(\mathrm{t}-1) \ldots \mathrm{y}(\mathrm{t}+1-\mathrm{s}) \mathrm{u}(\mathrm{t}-1) \ldots \mathrm{u}(\mathrm{t}+1-\mathrm{r})]^{\mathrm{T}}$ is a vector of the past plant and controller outputs, and $U(t)=[u(t) \ldots u(t+m-1)]^{T}$, is a vector of the future outputs of the controller. The matrices $\mathrm{P}, \mathrm{Q}$, and $\mathrm{R}$ are given below.

$$
P=\left[\begin{array}{ccc}
p_{11} & \cdots & p_{1(s+r-1)} \\
\vdots & & \vdots \\
p_{n_{2} 1} & \cdots & p_{n_{2}(s+r-1)}
\end{array}\right], \quad Q=\left[\begin{array}{cccc}
q_{11} & 0 & \cdots & 0 \\
q_{21} & q_{22} & \cdots & 0 \\
& \vdots & & \\
& & & \\
q_{m 1} & q_{m 2} & \cdots & q_{m m} \\
q_{n_{2} 1} & q_{n_{2}} & \cdots & q_{n_{2} m}
\end{array}\right], \quad R=\left[r_{1} \cdots r_{n_{2}}\right]^{T}
$$


The general aim of the predictive control scheme is that the future outputs on the considered horizon should follow a pre-determined reference trajectory and, at the same time, the necessary control effort should be minimized. A typical cost function includes increments of the control signal, the control signal itself or neither of them. Accordingly, it can be defined as follows [15]:

$$
J_{p r}=\sum_{i=1}^{n_{2}}\left[y_{p}(t+i)-y_{d}(t+i)\right]^{2}
$$

where $y_{d}(t)$ is the reference trajectory used over the prediction horizon. The optimal controller output is found by minimizing the above cost function such that.

$$
\frac{\partial J p r}{\partial u}=0
$$

Differentiating the cost function defined in equation (22), leads to the following optimal solution:

$$
U(t)=\left[Q^{T} Q\right]^{-1} Q^{T}[W(t)-P X(t)-\operatorname{Re}(t)]
$$

\section{SIMULATION RESULTS}

This simulation utilized the continuous muscle relaxation model depicted in Fig. $\mathbf{3}$. The initial condition was $0 \%$ relaxation and the set-point command signal was 0.80 (20\% EMG) for the first 100 minuets, 0.95 (5\% EMG) for the next 100 minuets, 0.90 (10\% EMG) for a further 50 minuets and finally $0.95(5 \%$ EMG) to the end of the simulation. The simulations were divided into the following three tasks.

\section{- Simulation Task-I: The Nominal Case}

The first task concerns with the controller performance when the plant has the nominal parameters $\left(K=1.0, \mathrm{~min}, T_{1}=4.81 \mathrm{~min}, T_{2}=34.42, T_{3}=3.08 \mathrm{~min}, T_{4}=10.64 \mathrm{~min}\right.$., $\tau=1 \min , \alpha=2.98$ and $\left.X_{\mathrm{E}}(50)=0.404\right)$.

\section{- Simulation Task-II: Nonlinearity mismatches}

The second task concerns with the controller performance when a nonlinear mismatch between the actual system's nonlinearity and those of the model occurs. Hence the model assumed a nonlinear Hill equation described by $\alpha=2.98$ and $X_{\mathrm{E}}(50)=0.404$, whereas the system had a nonlinear Hill equation described by $\alpha=4.0$ and $X_{\mathrm{E}}(50)=0.505$.

\section{- Simulation Task-III: Uncertainties}

Uncertainty can be seen as an existing nonlinearities in time variant processes whose dynamics are not fixed; unknown time delay and nonlinearities mismatch. The third task concerns the controller performance when the system was exhibiting large or unknown dead time and taking into account the nonlinearity mismatch. Hence, the time delay in equation (8) was increases to $4 \mathrm{~min}$ and the nonlinearity mismatch described in the second task was considered. 
Figure 7 depicts the controller response when the first task was considered. The control scheme governs the muscle relaxation process although the set-point command is near the saturation region of the system. Figure 8 depicts the performance of the proposed control scheme with existing the nonlinearity mismatch described in the second task. Finally, the performance of the control scheme with existing uncertainties described in the third task is depicted in Fig. 9. Simulation results show that the controller has efficient performances in spite of large delay time, nonlinearity mismatch and model uncertainties.

In order to clarify the vision, the performance of the proposed PSO-ARX scheme is compared with GPC algorithms described in [3] in the sense of ISE (integral of square error) and ITAE (integral time of the absolute error) defined in equations (26) and (27), respectively.

$$
\begin{aligned}
& I S E=\int_{t=0}^{\infty} e^{2}(t) \\
& I T A E=\int_{t=0}^{t=\infty} t|e(t)|
\end{aligned}
$$

where $e(\mathrm{t})$ are the error between the system output and the desired set-point .
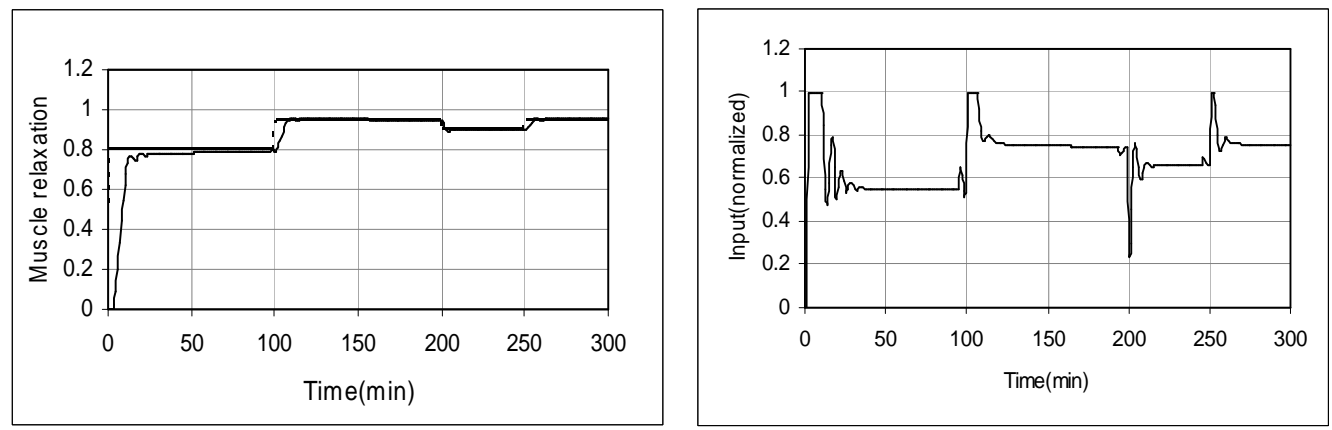

Fig. 7: The response of the muscle relaxation process using the proposed PSO-ARX (Simulation task-I).
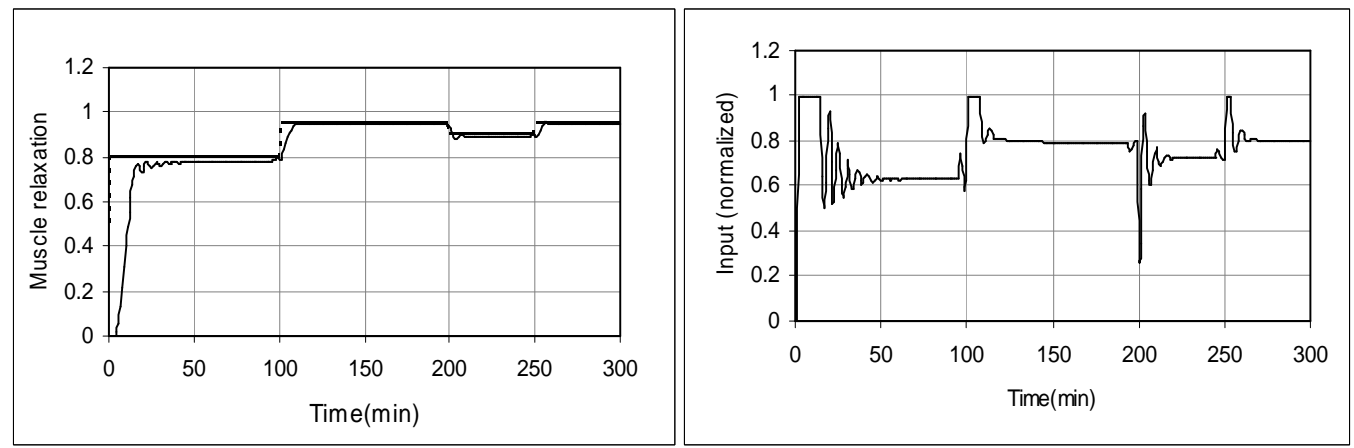

Fig. 8 : The response of the muscle relaxation process using the proposed PSO-ARX (Simulation task-II). 

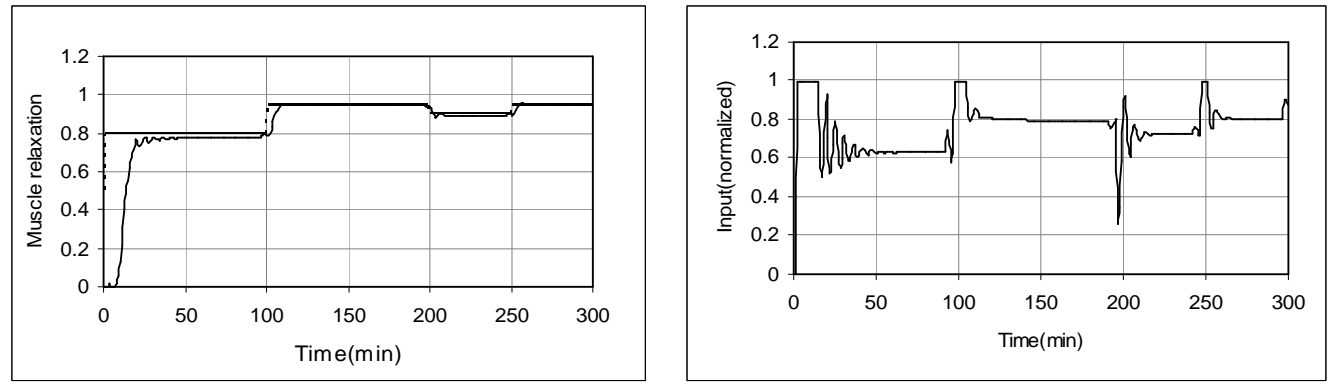

Fig. 9: The response of the muscle relaxation process using the proposed PSO-ARX (Simulation task-III).

Table 1 lists the ISE and ITAE values using the three algorithms with the first and the third simulation tasks. As shown in the table all ISE and ITAE values obtained with the proposed PSO-ARX are lower than those obtained using the GPC and NGPC algorithms respectively. For instant, the ITAE criteria, which tend to penalize responses for poor set-point tracking, are much lower with the new proposed controller than with the GPC and NGPC algorithms.

Table 1: A comparison between the proposed PSO-ARX controller scheme and the GPC schemes [3].

\begin{tabular}{|c|c|c|c|c|c|c|}
\hline \multirow{2}{*}{$\begin{array}{c}\text { Simulation } \\
\text { Task }\end{array}$} & \multicolumn{2}{|c|}{$\begin{array}{c}\text { The PSO-ARX } \\
\text { control scheme }\end{array}$} & \multicolumn{2}{c|}{ The GPC scheme } & \multicolumn{2}{c|}{ The NGPC scheme } \\
\cline { 2 - 7 } & ISE & ITAE & ISE & ITAE & ISE & ITAE \\
\hline The first task & 3.32 & 289.35 & 6.6 & 440 & 5.9 & 367 \\
\hline The third task & 4.63 & 463.43 & 8.9 & 954 & 9.0 & 663 \\
\hline
\end{tabular}

\section{CONCLUSIONS}

This paper proposed the PSO-ARX control scheme to deal with the following challenges: variation of time delay, nonlinearity mismatch, and uncertainty. These challenges were resulted from the large inter-individual and intra-individual variability of the patient's parameters. The basic idea behind the proposed algorithm is that its model structure can promptly be self-organized based on the challenges phased. Simulation results show that the proposed PSO-ARX control scheme is superior to the GPC and NGPC. They proved that the proposed scheme is a promising scheme for controlling more complex and multivariables medical systems. 


\section{REFERENCES}

[1] Geneilini, A. L., Feedback control of hypnosis and analgesia in humans, $\mathrm{PhD}$ thesis, Swiss Federal Institute of Technology (ETH), Zurich, Switzerland, March, 2001.

[2] Shieh, S. J., Linkens, A. D., and Peacock, E. J., " Hierarchical rule -based and self-organizing fuzzy logic control for depth of anaesthesia," IEEE Transaction on Systems, Man, and Cybernetics-Part C, vol. 29, no. 1, February, 1999.

[3] Mahfouf, M., and Linkens, A. D., Generalized predictive control and bioengineering, T. J. International Ltd, Padstow, UK, 1998.

[4] Brown, B. H., Asbury, Linkens, A. D., Perks, P., and Anthony, M., "Closed-loop control of muscle relaxation during surgery," Clinical Physics and Physiological Measurement, vol. 1, pp. 203-210, 1980.

[5] Smith O. J. M., "A controller to overcome dead-time," Journal of the Instrument, Society of America, vol. 6, pp. 23-33, 1959.

[6] Linkens, A. D., Mahfouf, M., and Asbury, A. J., "Generalized predictive control applied to muscle relaxant anaesthesia," IEE Conference on Adaptive Control, Edinburgh, UK, March, 1991.

[7] Ward, S., Neill, E. A. M., Weatherley, B. C., and Corall, I. M., "Pharmacokinetics of atracurium besylate in healthy patients (after a single i.v bolus dose)," British Journal of Anaesthesia, vol. 55, 1983.

[8] Weatherley, B. C., Wiliams, S. G., and Neill, E. A. M., "Pharmacokinetics, Pharmacodynamics and dose response relationship of atracurium administrated i.v. ," British Journal of Anaesthesia, vol. 55, 1983.

[9] Whiting, B., and Kelman, A. W., "The modeling of drug response," Clinical Science, vol. 59, 1980.

[10] Nabila M. El-Rabaie and Tarek A. Mahmoud, "Multivariable dynamic systems modeling and control using a new particle swarm algorithm-local model network, "Journal of Engineering Science, JES'06, Faculty of Engineering, Assuit Unv., Egypt, vol. 34, no. 2, March, 2006.

[11] Takagi, T., and Sugeno, M., "Fuzzy identification of systems and its applications to modeling and control," IEEE Transactions on Systems, Man, and Cybernetics, vol. SMC-15, 1985.

[12] Zhang. Q., and Benveniste, A., "Wavelet networks," IEEE Transactions on Neural Networks, vol. 3, no. 6, 1992.

[13] Larsen P. M., "Industrial applications of fuzzy logic control," Int. J. Man Mach. Studies, vol. 22, 1980.

[14] Carpenter, G.A., Grossberg, S., and Rosen, D.B., "Fuzzy ART: Fast stable learning and categorization of analogue patterns by an adaptive resonance system," Neural Networks, vol. 4, 1991.

[15] Linkens, D.A., and Kandiah S., "Long- range predictive control using fuzzy process model," Trans IChemE, vol.74, Part A, 1996. 


\section{نظام ذاتي التكوين للتحمم في عملية استرخاء العضلات}

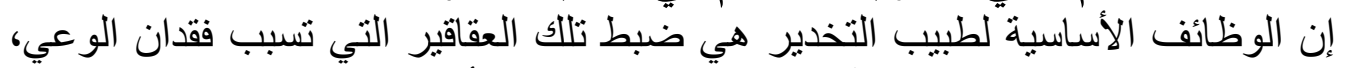

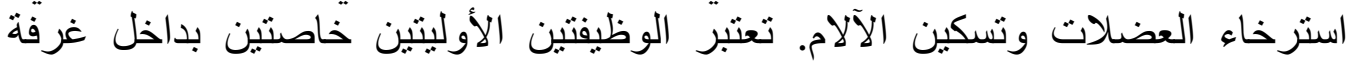

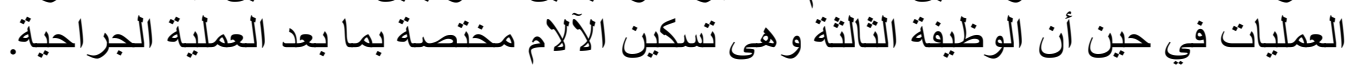

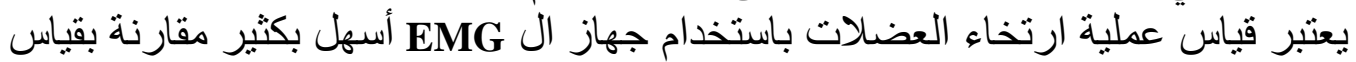

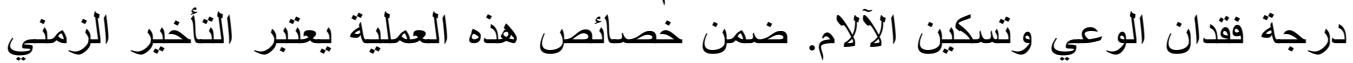

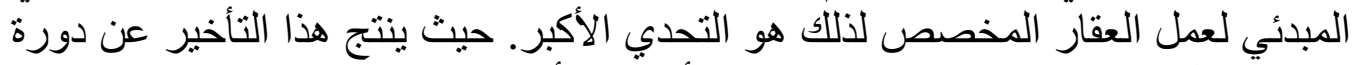

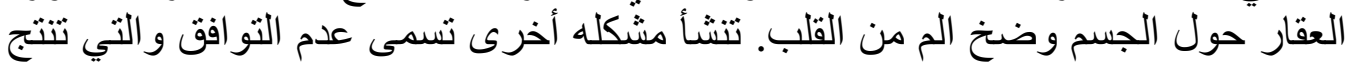

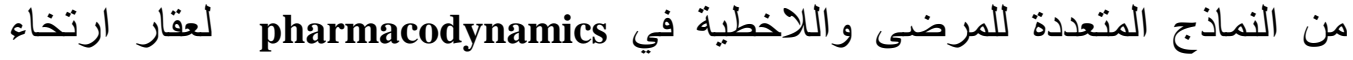

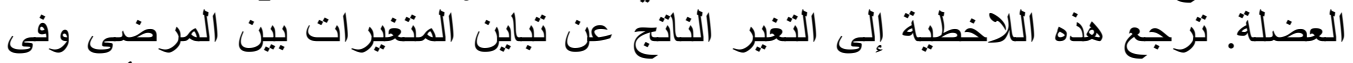

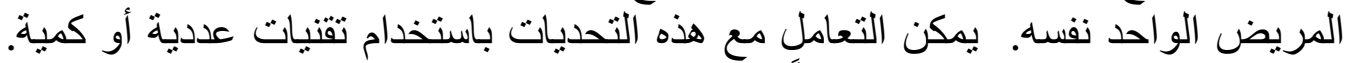

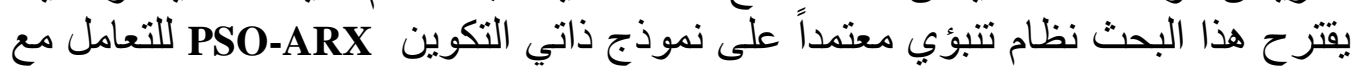

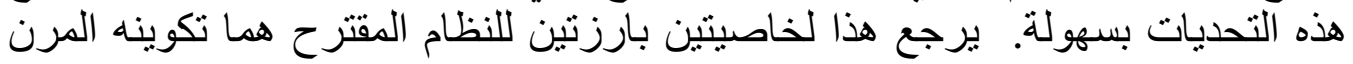

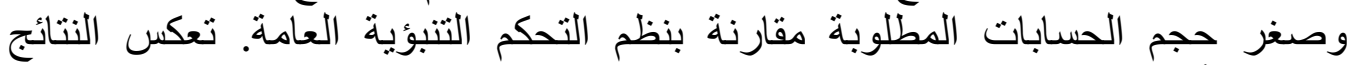
المحاكية أفضلية النظام المقتر ح بالنسبة لتلك النظم. 